\title{
Coatings Probed by Positrons
}

B. Nielsen, L.D. Hulett Jr.*, S. Wallace*, J. Xu*, C.S. Szeles, K.G. Lynn and J. Pfau**

Brookhaven National Laboratory

* Oak Ridge National Laboratory

** Masonite Division, International Paper Co.

Thin film coatings such as TiN and polymers (paint) have been studied using positrons. Positrons provide information on defect structure. It is shown how the positron response is related to properties of the coatings such as stoichiometry. Further positrons provide a measure of the uniformity of the coating. Preliminary results on the effect of weathering (ultraviolet exposure) of polymer protective coatings are very encouraging.

\section{Introduction.}

A variety of modern technologies, ranging from protective coatings to silicon technology, involve thin film structures. The important role extended defects play in the quality of such structures has been extensively documented by studies using transmission electron microscopy. The development of variable energy positron beams (VEP) has provided a non destructive probe for submicroscopic defects in thin films and at interfaces.For a review see ref. 1 . This paper presents results from two recent studies of protective coatings, TiN and polymer binder (paint), to illustrate the potential of VEP. Defect structures were investigated as a function of composition in TiN films fabricated by sputter deposition and also the recovery of defects during annealing was studied. This provides examples of defect evolution during synthesis and processing. Early stages of degradation was detected in polymer binders exposed to weathering effects (solar radiation and water spray). A more detailed presentation of studies of TiN films has been given in ref 2. and for polymer binders in ref. 3.

\section{Depth resolved positron annihilation spectroscopy.}

Variable energy positron beams provide a nondestructive probe for depth profiling of open volume defect structures, such as vacancies and voids and in certain cases impurity related defects. The mean penetration depth of the positron is varied simply by tuning the beam energy. After the positron is implanted and thermalized, it diffuses, possibly traps at a defect, and subsequently annihilates with an electron, emitting two 
$\gamma$-quanta. These quanta carry information about the site of annihilation, which can be extracted in various ways. One, which has been used in this work is to measure the Doppler broadening of the annihilation radiation (DBAR), caused by the momentum of the annihilating electron-positron pair. It is the strong variation in this momentum from perfect to defect lattice sites that provides the base of the technique. The broadening is measured with an energy sensitive gamma-ray detector. The shape of the spectrum is characterized by a single parameter, $s$, the lineshape parameter, defined as the area of a fixed region in the center of the annihilation peak divided by the total area of the peak. In general an increase in $S$ corresponds to an increase in defect concentration and/ or defect size. By scanning the incident beam energy and recording separate annihilation spectra for each energy, it is possible to obtain values of $s$ that are characteristic of different depths of the sample.

\section{Defect structure of sputter deposited TiN.}

\section{Experimental procedure}

The TiN samples were prepared by reactive d.c. magnetron sputtering. The details of their preparation are described elsewhere (2). Film compositions have been determined by the electron micro-probe method. Films of $\operatorname{TiN}_{x}$ were prepared at various compositions: stoichiometric $(x=1.00), N$ rich $(x=1.07)$ and finally a Ti rich $(x=.72)$. $x-r a y$ measurements indicated that all films are single phase. All films were grown to a thickness of approximately $1.5 \mathrm{um}$ on stainless steel substrates.

\section{Results and discussion.}

The result of the positron energy/ depth scan of $s$ through the as received films are shown in fig 1 . The $S$ values corresponding to the energy range from $0-20 \mathrm{keV}$ are related to the defect structure of the TiN thin films. It can be seen that the stoichiometric film appears to be less defective (i.e. have lower s-values) than either of the two other films. As the implantation energy is increased a significant fraction of the positrons is implanted into the substrate; thus, the $S$ values at high energies reflect the condition of the stainless steel substrate rather than that of the film. The defect concentration in the super- and substoichiometric films is sufficiently high to cause saturation trapping, i.e. all positrons are trapped. The s-parameter does in this condition not reveal information on the defect concentration but only on the defect structure. It is clear that the defect structure of the N-rich film is substantially different from that of the Ti-rich film. It is also clear that the defect structure in the N-rich sample shows a variation in depth. Measurements of the $S-$ parameter as a function of energy/depth is shown in fig 2 for the stoichiometric film as received and after a 1 hour anneal at $900^{\circ} \mathrm{C}$. As seen the s-parameter generally shows a lowering after annealing, i.e. a reduction in the average defect size. However, the lowering is not uniform. It is mainly confined to a region in the middle of the film. The lowering near the stainless steel interface is less pronounced and in a region near the surface there is actually an increase in $S$. We speculate that this is due to outdiffusion of nitrogen. A clear defect recovery is also observed in the stainless steel substrate. This illustrates a unique capability of the VEP technique to be able to follow simultaneously defect evolution in film and substrate. The sensitivity to the interface is related to the positron transport to the 


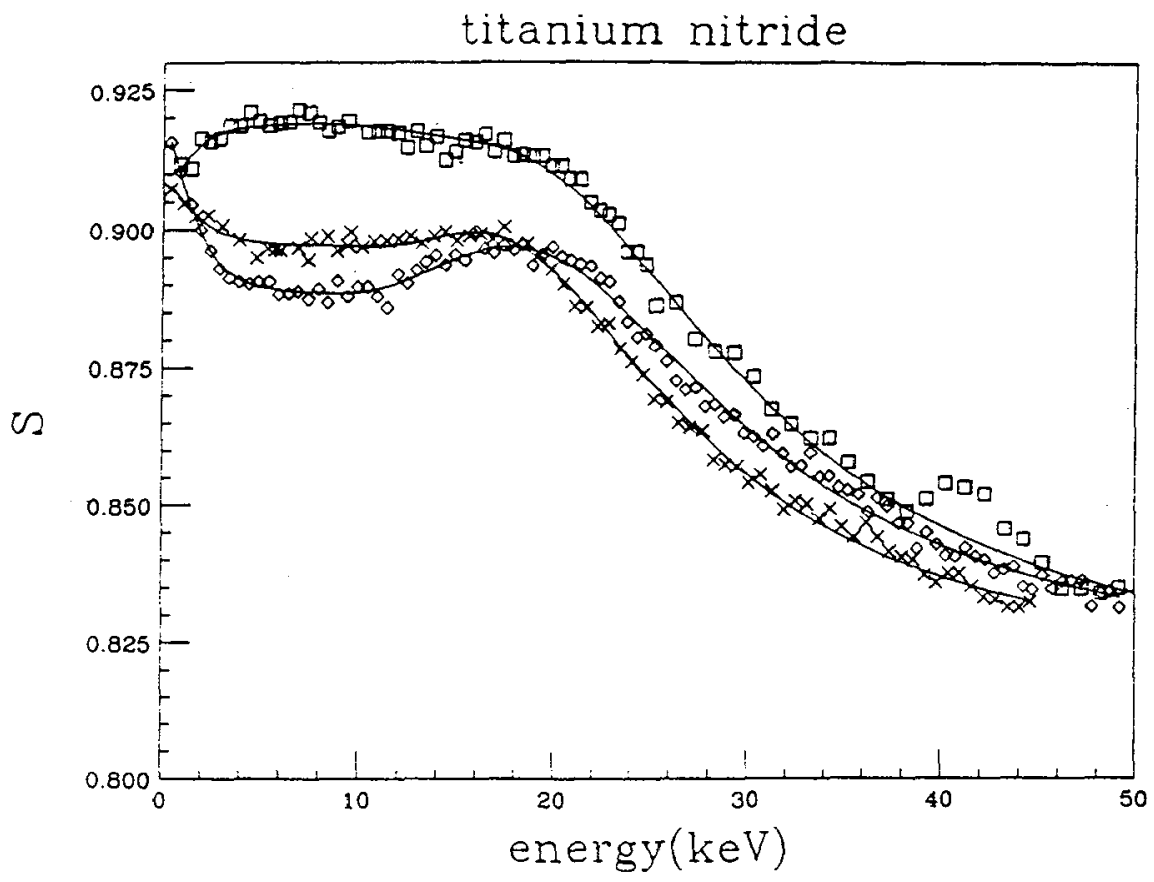

Figure 1 Shown is the relative s-paramter values as a function of incident positron energy/ depth for $\mathrm{TiN}_{\mathrm{x}}$ samples of various composition $x$. Stoichiometric $0, N$ rich $x$ and $\mathrm{Ti}$ rich $a$

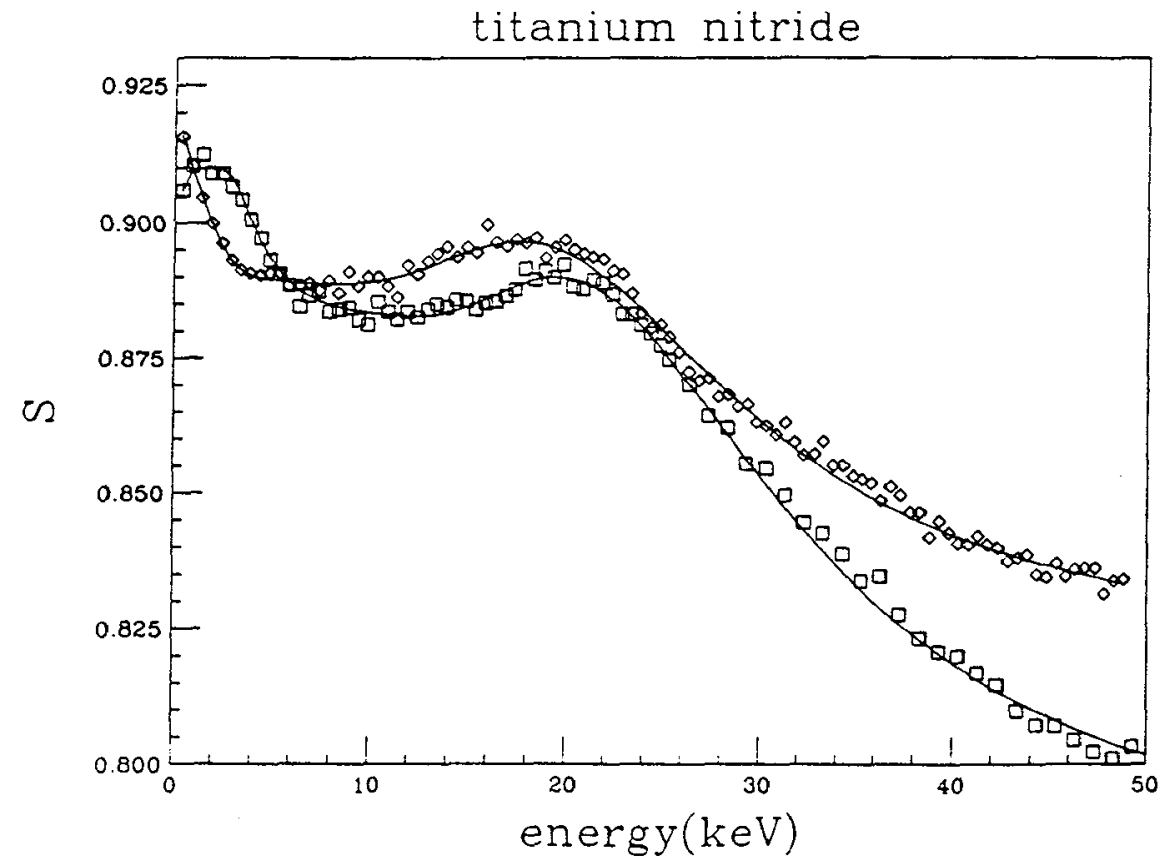

Figure 2 shown is the relative $s$-parameter values as a function of incident energy/ depth for a stoichiometric TiN film on stainless steel as received and after annealing at $900^{\circ} \mathrm{C}$ a 
interface region, i.e. the positron diffusion length in the film and substrate scaled to the in-verse depth of the interface. In this case where the diffusion length is very short (of the order 10nm) in the TiN and stainles steel and the interface is located relatively deep, the senstivity is low. It has been demonstrated that for thinner fims and greater diffusion length in the substrate that a very high sensitivity to the interface can be achieved (4).

\section{Weathering effects on protective polymer coatings.}

\section{Experimental procedure.}

Water based paints of styrene-acrylic polymer binder have been studied. They were applied as 25 - 40 micrometer films on aluminum substrates. Panels containing binder only, and panels containing binder plus $40 \%$ $\mathrm{TiO}_{2}$ pigment volume content were prepared. The panels were oven dried at $165^{\circ} \mathrm{C}$ for 10 minutes. The panels were weathered at the EMMAQUA facility. This device subjects the specimens to concentrated sunlight reflected from an array of mirrors and to periodic water spraying.

\section{Results and discussion.}

Figure 3 shows $S$ vs energy/ depth scans for styrene-acrylic binder films. These films had no $\mathrm{TiO}_{2}$ pigments, being completely transparent. It is seen that at all energies/ depths. The S-parameters for the 15 days weathered film are lower than those of the non-weathered specimen. It is also observed that the s-parameter increases with depth into the film. Figure 4 compares $S$ vs energy scans for films that contain $40 \% \mathrm{TiO}_{2}$ pigment. It is seen that the weathering has caused the s-parameter to decrease for this specimen also. Note that for low energies of positron implantation, less than 500eV, the s-parameter for samples with and without pigments are about the same, but for energies above about $1 \mathrm{keV}$ the s-parameters decrease sharply in the pigment containing samples. This suggests that the outermost surfaces of the films are depleted in $\mathrm{TiO}_{2}$ pigment and that the $s$ parameters are lower at greater depths in the film due to the pigment. In all cases the films show a clear lowering of the $s$ parameter after weathering. Under well-defined cicumstances, a lowering of $S$ i.e. broadening of the $511 \mathrm{keV}$ annihilation line can be ascribed to reductions in free volume contents of polymers. Such is the case with the work of Jean and Deng $(5,6)$, where free volume content was altered by physical processes (temperature and pressure changes). For this work, however, changes in the Doppler broadening spectra may be due to more complex factors, such as changes due to the formation of free radicals. More detailed future work is needed to reveal this.

\section{Conclusion.}

In studies of TiN films it is shown that the defect strucure varies significantly with composition. A stoichiometric prepared film is less defective than Ti rich or $\mathrm{N}$ rich films, however, the defect structure shows a variation in depth. Annealing causes a clear defect recovery in the middle of the film, less near the interface to the stainless steel substrate and actually an increase in the near surface region. It is observed that the annealing causes a clear defect recovery in the stainless steel. The positron based technique has been used to detect early onset of weathering effects on the properties of protective polymer films. This high sensitivity to changes in early stages of degradation has the potential to contribute to the durability of polymer coatings. 


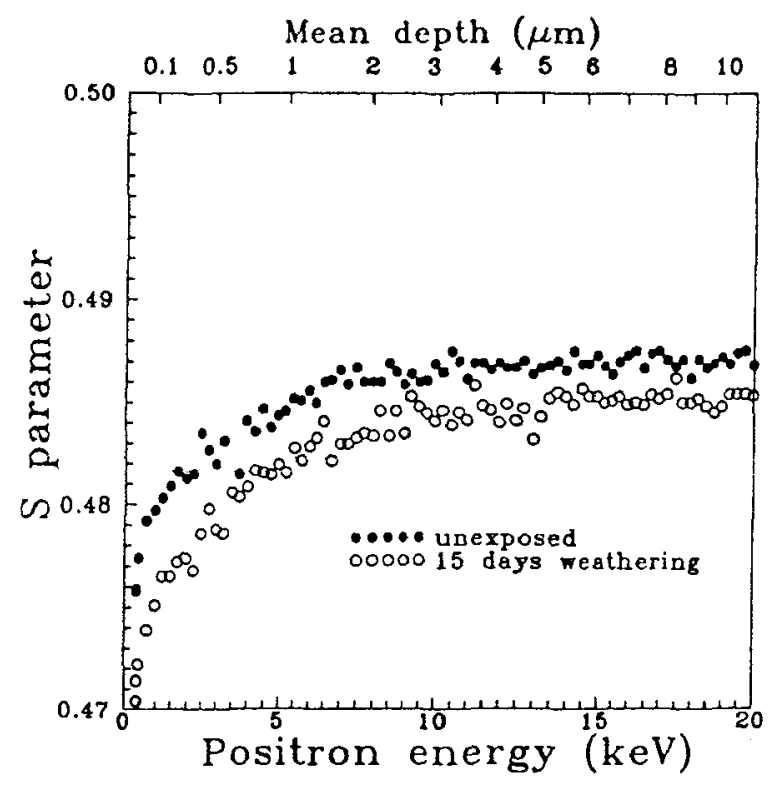

Figure 3 The effect of weathering on relative s-parameters of waterbased styrene-acrylic paint binder films as a function of incident positron energy/ depth. Films were visually transparent, with no $\mathrm{TiO}_{2}$ pigment.

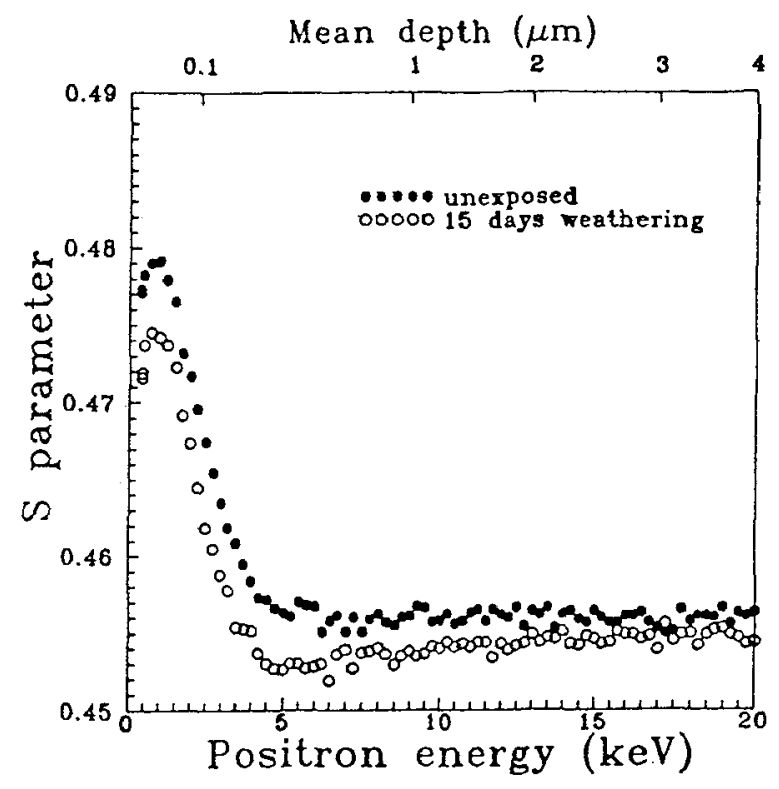

Figure 4 The effect of weathering on relative s-parameter values of water-based styrene-acrylic paint films, having $40 \% \mathrm{TiO}_{2}$ pigment, as a function of incident positron energy/ depth. 


\section{References}

[1] P.J. Schultz and K.G. Lynn, Rev. Mod. Phys., 60, 701 (1988)

[2] J.P. Schaffer, A.B. De Wald, Jr., R.L. Frost, A.J. Perry, B. Nielsen and K.G. Lynn, Surf. Coat. Technol. 36, 593 (1988)

[3] L.D. Hullett, Jr., S. Wallace, J. Xu, B. Nielsen, Cs. Szeles, K.G. Lynn, J. Pfau and A. Schaub, Appl. Surf. Sci. (in press)

[4] T.C. Leung, P. Asoka-Kumar, B. Nielsen and K.G. Lynn, J. Appl. Phys. 73, 168 (1993)

[5] Y.C. Jean, Microchemical J. 42, 72 (1990)

[6] Y.C. Jean and Q. Deng, J. Polymer Sci. B., Polymer Phys., 29, 1359 (1992) 